\title{
Common Fixed Points for Suzuki-Generalized Nonexpansive Maps
}

\author{
Maryam A. Alghamdi, ${ }^{1}$ Sompong Dhompongsa, ${ }^{2}$ and Naseer Shahzad ${ }^{3}$ \\ ${ }^{1}$ Department of Mathematics, Science Faculty for Girls, King Abdulaziz University, P.O. Box 4087, Jeddah 21491, Saudi Arabia \\ ${ }^{2}$ Department of Mathematics, Faculty of Science, Chiang Mai University, Chiang Mai 50200, Thailand \\ ${ }^{3}$ Department of Mathematics, King Abdulaziz University, P.O. Box 80203, Jeddah 21859, Saudi Arabia
}

Correspondence should be addressed to Naseer Shahzad; nshahzad@kau.edu.sa

Received 17 May 2013; Revised 27 July 2013; Accepted 27 July 2013

Academic Editor: E. Karapinar

Copyright (C) 2013 Maryam A. Alghamdi et al. This is an open access article distributed under the Creative Commons Attribution License, which permits unrestricted use, distribution, and reproduction in any medium, provided the original work is properly cited.

A common fixed point theorem for a pair of maps satisfying condition $(\mathrm{C})$ is proved under certain conditions. We extend the wellknownDeMarr's fixed point theorem to the case of noncommuting family of maps satisfying condition (C). As for an application, an invariant approximation theorem is also derived.

\section{Introduction}

Jungck [1] initiated the systematic study of finding a common fixed point of a pair of commuting maps. This problem of finding a common fixed point has been of significant interest in the area of fixed point theory and has been studied by many authors such as in [2-6]. At the first time, the commutativity for two maps was always assumed to find a common fixed point. Later, it was found that the two maps were not necessarily commutative at each point, and then weaker forms of commutativity were defined to obtain a common fixed point for maps on a metric space. For example, the notions of weakly commutative maps [2], compatible maps (weakly compatible maps) [7], biased maps [8], $R$-subweakly commuting maps [4], and occasionally weakly compatible [9] have been introduced and used to find common fixed points of maps.

Recently, Chen and Li [5] introduced the class of Banach operator pairs and, in [10], they investigated the common fixed point problem for nonexpansive maps where $(I, T)$ is a Banach operator pair. Also, they extended the well-known De Marr's fixed point theorem to the noncommuting case.

More recently, Suzuki [11] introduced a condition on maps, called condition (C) (maps satisfying condition (C) are also known as Suzuki-generalized nonexpansive maps), and obtained some fixed point theorems and convergence theorems for such maps. Dhompongsa et al. [12] and Dhompongsa and Kaewcharoen [13] made significant contribution to fixed point theory for maps satisfying condition (C). For more results see [14].

In this paper, we discuss a common fixed point problem for a Banach operator pair satisfying condition (C). A family of maps satisfying condition $(\mathrm{C})$ is also investigated. As for an application, an invariant approximation theorem is obtained.

\section{Preliminaries}

Let $E$ be a Banach space. $E$ is said to be

(i) strictly convex if $\|x+y\|<2$ for all $x, y \in E$ with $\|x\|=\|y\|=1$ and $x \neq y$,

(ii) uniformly convex in every direction (UCED) if, for $\varepsilon \in(0,2]$ and $z \in E$ with $\|z\|=1$, there exists $\delta(\varepsilon, z)>$ 0 such that

$$
\|x+y\| \leq 2 \quad(1-\delta(\varepsilon, z))
$$

for all $x, y \in E$ with $\|x\| \leq 1,\|y\| \leq 1$ and $x-y \in\{t z$ : $t \in[-2,-\varepsilon] \cup[+\mathcal{E},+2]\}$.

It is obvious that being UCED implies strict convexity. 
Let $K$ be a nonempty subset of $E$ and let $T$ be a self-map of $K$. We denote by $F(T)$ the set of fixed points of $T$; that is, $F(T)=\{x \in K: T x=x\}$. Also, if $I$ and $T$ are self-maps of $K$, we denote by $F(I, T)$ the set of common fixed points of $I$ and $T$; that is, $F(I, T)=\{x \in K: I x=T x=x\}$. If $H$ is a nonempty family of self-maps of $E$, a point $x \in E$ is called common fixed point of $H$ if it is the fixed point of each member of $H$.

The map $T$ is called

(i) nonexpansive if

$$
\|T x-T y\| \leq\|x-y\|, \quad \forall x, y \in K,
$$

(ii) quasi-nonexpansive if $F(T) \neq \phi$ and

$$
\|T x-p\| \leq\|x-p\|, \quad \text { for } x \in K, p \in F(T) .
$$

Suzuki [11] introduced a condition on maps, called condition (C), which is weaker than nonexpansiveness and stronger than quasi-nonexpansiveness.

Definition 1 (see [11]). A self-map $T$ of $K$ is said to satisfy condition $(C)$ if

$$
\frac{1}{2}\|x-T x\| \leq\|x-y\| \text { implies }\|T x-T y\| \leq\|x-y\|
$$

for all $x, y \in K$.

Example 2 (see [13]). Define a map $T$ on $[0,3(1 / 2)]$ by

$$
T x= \begin{cases}0 & \text { if } x \in[0,3] \\ 4 x-12 & \text { if } x \in\left[3,3 \frac{1}{4}\right] \\ -4 x+14 & \text { if } x \in\left[3 \frac{1}{4}, 3 \frac{1}{2}\right] .\end{cases}
$$

Then $T$ is a continuous map satisfying condition $(C)$ and $T$ is not nonexpansive.

Proposition 3 (see [11]). Let $K$ be a nonempty subset of a Banach space E. Assume that $T: K \rightarrow K$ is a nonexpansive map. Then $T$ satisfies condition $(C)$.

Proposition 4 (see [11]). Let $K$ be a nonempty subset of a Banach space E. Assume that a map $T: K \rightarrow K$ satisfies condition $(C)$ and has a fixed point. Then $T$ is a quasinonexpansive map. pairs.

Chen and $\mathrm{Li}$ [5] introduced the class of Banach operator

Definition 5 (see [5]). Let $(X, d)$ be a metric space; the pair $(I, T)$ of two self-maps $I$ and $T$ of $X$ is called a Banach operator pair if the set $F(T)$ of fixed points of $T$ is $I$-invariant; that is, $I(F(T)) \subseteq F(T)$.

A Banach operator pair $(I, T)$ depends on the order of $I$ and $T$; that is, if $(I, T)$ is a Banach operator pair, $(T, I)$ need not be such a pair. It is well known that for two self-maps $I$ and $T$ of a metric space $X$, the pair $(I, T)$ is a Banach pair if and only if $I$ and $T$ commute on the set $F(T)$ [5].
Example 6 (see [5]). Let $f$ and $g$ be two self-maps of $X=\mathbb{R}^{2}$ defined by

$$
\begin{gathered}
f(s, t)=\left(s^{2}+t^{2}+s-1, s^{2}+t^{2}+t-1\right), \\
g(s, t)=\left((s-t)^{2}+2 s-t,(s-t)^{2}+s\right)
\end{gathered}
$$

for $(s, t) \in \mathbb{R}^{2}$. Directly, we have

$$
\begin{gathered}
F(f)=\left\{(s, t) \in \mathbb{R}^{2}: s^{2}+t^{2}-1=0\right\}, \\
F(g)=\left\{(s, t) \in \mathbb{R}^{2}: s-t=0 \text { or } s-t+1=0\right\} .
\end{gathered}
$$

The following assertions can be verified:

(i) $f(F(g)) \subseteq F(g)$, and hence $(f, g)$ is a Banach operator pair on $\mathbb{R}^{2}$; equivalently, $f$ and $g$ commute on the set $F(g)$.

(ii) $(g, f)$ is not a Banach operator pair, since for $(1,0) \epsilon$ $F(f), g(1,0)=(3,2)$ is not in $F(f)$.

The following proposition for Banach operator pairs can be found in [10].

Proposition 7. If $F(T)$ is a q-star shaped set (i.e., $t x+(1-t) q \in$ $F(T)$ for any $x \in F(T)$ and $0 \leq t \leq 1)$ with $q \in F(T)$, then $(I, T)$ is a Banach operator pair if and only if the pairs $\left(I_{k}, T\right)^{\prime} s$ are Banach operator pairs for all $k \in[0,1]$, where $I_{k} x=(1-$ k) $I x+k q$.

Definition 8 (see [10]). Let $T$ and $I$ be two self-maps of a metric space $X$. The pair $(I, T)$ is called symmetric Banach operator pair if both $(T, I)$ and $(I, T)$ are Banach operator pairs; that is, $T(F(I)) \subset F(I)$ and $I(F(T)) \subset F(T)$.

The pair $(I, T)$ is a symmetric Banach operator pair if and only if $T$ and $I$ are commuting on $F(T) \cup F(I)$. It is easy to see that a Banach operator pair may not be a symmetric Banach operator pair; see [10].

Definition 9 (see [10]). Let $H$ be a nonempty family of selfmaps of a metric space $X . H$ is called a Banach operator family if for all $I, T \in H,(I, T)$ is a symmetrical Banach operator pair.

In 1963, DeMarr [15] stated the following well-known fixed point theorem for a family of commuting nonexpansive maps.

Theorem 10 (DeMarr [15]). If $K$ is a nonempty compact convex subset of a Banach space $X$ and $H$ is a nonempty family of commuting nonexpansive maps of $K$ into itself, then the family $H$ has a common fixed point in $K$.

Recently Chen and Li [10] extended DeMarr's theorem to the noncommuting case.

Theorem 11 (see [10]). Let $K$ be a nonempty closed convex subset of a normed space $E$ and let $H$ be a nonempty family of nonexpansive maps of $K$ into itself. If $H$ is a Banach operator family and there exists a $T \in H$ such that $\overline{T(K)}$ is compact, then $H$ has a common fixed point in $K$. 
We now collect some results about condition (C) which will be used in the sequel.

Lemma 12 (see [11]). Let $K$ be a nonempty closed subset of a Banach space E. Assume that $T: K \rightarrow K$ satisfies condition (C). Then $F(T)$ is closed. Moreover, if $E$ is strictly convex and $K$ is convex, then $F(T)$ is also convex.

Theorem 13 (see [14]). Let $K$ be a closed bounded convex subset of a Banach space E. Assume that $T: K \rightarrow K$ is a map satisfying condition $(C)$ and that $\overline{T(K)}$ is compact. Then $T$ has a fixed point.

Lemma 14 (see [11]). Let $K$ be a nonempty subset of a Banach space E. Assume that $T: K \rightarrow K$ is a map satisfying condition (C). Then for $x, y \in K$, the following hold:

(i) $\left\|T x-T^{2} x\right\| \leq\|x-T x\|$,

(ii) either $\frac{1}{2}\|x-T x\| \leq\|x-y\|$ or $\frac{1}{2}\left\|T x-T^{2} x\right\| \leq\|T x-y\|$ holds,

(iii) either $\|T x-T y\| \leq\|x-y\|$ or $\left\|T^{2} x-T y\right\| \leq\|T x-y\|$ holds.

\section{Main Results}

Lemma 15 (see [16] or [15]). Let $M$ be a nonempty compact subset of a Banach space E. Let $\delta$ be the diameter of $M$. If $\delta>0$, then there exists an element $u \in \overline{c o} M$ such that

$$
\sup \{\|x-u\|: x \in M\}<\delta,
$$

where $\overline{c o} M$ is the smallest closed convex set containing $M$.

Following [15], we are able to prove the following lemma.

Lemma 16. Let $K$ be a nonempty closed convex subset of a Banach space E. Suppose that $T: K \rightarrow K$ satisfies condition (C) such that there exists a compact set $M \subset F(T)$ not reduced to a point. Then there exists a nonempty closed convex set $K_{1}$ such that

(1) $K_{1} \subset K$ and $T\left(K_{1}\right) \subset K_{1}$,

(2) $M \cap\left(K_{1}\right)^{c} \neq \emptyset$.

Proof. Let $\delta$ be the diameter of $M$. Since $M$ is not reduced to a point, we have $\delta>0$. According to Lemma 15, there is $u \in \overline{c o} M$ such that

$$
\delta_{1}=\sup \{\|x-u\|: x \in M\}<\delta .
$$

For each $x \in M$, define

$$
U(x)=\left\{y:\|y-x\| \leq \delta_{1}\right\} .
$$

Since $u \in U(x)$ for each $x \in M$, it follows that $K_{0}=$ $\bigcap_{x \in M} U(x) \neq \emptyset$. It is easy to check that $K_{0}$ is closed and convex. Let $K_{1}=K_{0} \cap K$. Then $K_{1}$ is not empty since $u \in K_{1}$. For any $x \in K_{1}$ and any $z \in M$, we have $x \in U(z)$; that is, $\|x-z\| \leq \delta_{1}$. Since

$$
\frac{1}{2}\|z-T z\|=0 \leq\|z-x\|,
$$

we obtain that

$$
\|z-T(x)\|=\|T(z)-T(x)\| \leq\|z-x\| \leq \delta_{1} .
$$

That is, $T(x) \in U(z)$. This is true for any $z \in M$; thus $T(x) \in$ $K_{1}$. This shows that $T(x) \in K_{1}$ for all $x \in K_{1}$. Recalling that $M$ is compact, therefore, there exist $x_{0}, x_{1} \in M$ such that $\| x_{0}-$ $x_{1} \|=\delta>\delta_{1}$. Thus, $x_{1} \notin U\left(x_{0}\right) \supset K_{1}$; that is, $x_{1} \in M \cap$ $\left(K_{1}\right)^{c} \neq \emptyset$.

Theorem 17. Let $K$ be a nonempty closed bounded convex subset of a Banach space E. Suppose that $T$ and $I$ are two self-maps on $K$ satisfying condition $(C)$. If $(I, T)$ is a Banach operator pair, $I$ is nonexpansive, and $\overline{T(K)}$ is compact, then $F(I, T) \neq \emptyset$.

Proof. Let $\Gamma$ be the set of all nonempty closed bounded convex subsets $A$ of $K$ such that $T(A) \subset A$ and $I(A) \subset A$ and $\overline{T(A)}$ is compact. Since $K \in \Gamma$, then $\Gamma$ is nonempty. Define a partial order " $\leq$ " by set inclusion on the set $\Gamma$; that is, $A_{i} \leq A_{j}$ whenever $A_{i} \subseteq A_{j}$.

Let $\Gamma_{0}$ be any total ordering subset of $\Gamma$ and $A \in \Gamma_{0}$. Since $A$ is closed, we have $\overline{T(A)} \subset A$, and since $\overline{T(A)}$ is compact, it follows that

$$
\emptyset \neq \bigcap_{A \in \Gamma_{0}} \overline{T(A)} \subset \bigcap_{A \in \Gamma_{0}} A=A_{0} .
$$

It is clear that $A_{0} \in \Gamma$. By Zorn's lemma, $\Gamma$ has a minimal set $K_{0}$.

Since $T$ satisfies condition (C) and $\overline{T\left(K_{0}\right)}$ is compact, then, by Theorem 13, T has a nonempty fixed point set $F(T) \subset$ $K_{0}$. It follows that $F(T)$ is a closed subset of $\overline{T\left(K_{0}\right)}$ and thus is compact. On the other hand, we have $T(F(T))=F(T)$, and since $(I, T)$ is a Banach operator pair, it implies that $I(F(T)) \subset F(T)$. Using Zorn's lemma again, there exists a minimal nonempty compact subset $M$ of $F(T)$ which satisfies $T(M)=M$ and $I(M) \subseteq M$ ( $M$ is not necessarily convex $)$.

Next, we show $I(M)=M$. If $I(M) \neq M$, then we have $I(I(M)) \subset I(M)$, and $I(M)$ is compact because $I$ is continuous. Also, we have $T(I(M))=I(M)$ since $I(M) \subset M \subset F(T)$. This contradicts the minimality of $M$.

If $M$ has only one point, the proof is finished. Suppose that $M$ has at least two points. By Lemma 16 there exists a set $K_{1}$ satisfying $T\left(K_{1}\right) \subset K_{1}$ and $M \cap\left(K_{1}\right)^{c} \neq \emptyset$. Since $I$ is nonexpansive and $I(M)=M$, it follows that $K_{1} \in \Gamma$ which implies that $K_{1}$ is a proper subset of $K_{0}$ and this contradicts the minimality of $K_{0}$. This completes the proof.

Theorem 18. Let $K$ be a nonempty closed bounded convex subset of a strictly convex space E. Suppose that $T$ and I are two self-maps on $K$ satisfying condition $(C)$. If $(I, T)$ is a Banach operator pair and $\overline{T(K)}$ is compact, then $F(I, T) \neq \emptyset$. 
Proof. By Theorem 13 and Lemma 12, F(T) is a nonempty closed bounded convex set. It is compact since $\overline{T(K)}$ is compact. Since $I(F(T)) \subset F(T)$, again by Theorem $13, I$ has a fixed point in $F(T)$; that is, $F(T) \cap F(I) \neq \emptyset$.

Corollary 19. Let $K$ be a nonempty closed bounded convex subset of an UCED Banach space E. Suppose that $T$ and $I$ are two self-maps on $K$ satisfying condition $(C)$. If $(I, T)$ is a Banach operator pair and $\overline{T(K)}$ is compact, then $F(I, T) \neq \emptyset$.

Example 20. Consider $\mathbb{R}$ with the usual metric and let $K=$ $[0,3(1 / 2)]$. Define a map $T$ on $K$ by

$$
T x= \begin{cases}0 & \text { if } x \in[0,3] \\ 4 x-12 & \text { if } x \in\left[3,3 \frac{1}{4}\right] \\ -4 x+14 & \text { if } x \in\left[3 \frac{1}{4}, 3 \frac{1}{2}\right]\end{cases}
$$

and define a map $I$ on $K$ by $I x=x^{2} / 7$. Then $T$ is a continuous map satisfying condition $(C)$ and $T$ is not nonexpansive (see [13]) and $I$ is nonexpansive and hence satisfies condition $(C)$. Also $(I, T)$ is a Banach operator pair. Therefore, all conditions of Theorem 17 (and Theorem 18) are satisfied and $I$ and $T$ have a common fixed point. Note that Theorem 2.1 in Chen and $\mathrm{Li}$ [10] is not applicable here.

Next, we show a common fixed point theorem of a countable family of maps satisfying condition (C). We need first the following proposition which shows that for a given map $I$ there are a lot of maps $T$ such that $(I, T)$ is a symmetric Banach operator pair.

Proposition 21 (see [10]). Let I be a self-map on a convex subset $K$ of a normed space $E$ and let $\alpha$ be a map from $K$ to $[0,1]$ such that the set $\{x \in X: \alpha(x)=0\}$ is I-invariant; that is, $\alpha(I x)=0, \forall x \in\{x \in X: \alpha(x)=0\}$. Define

$$
T_{\alpha} x=\alpha(x) I x+(1-\alpha(x)) x .
$$

Then $\left(I, T_{\alpha}\right)$ is a symmetric Banach operator pair.

Theorem 22. Let $K$ be a nonempty closed bounded convex subset of a Banach space E. Suppose that $H$ is a nonempty family of self-maps on $K$ satisfying condition (C). If $H$ is a Banach operator family and there exists a $T_{1} \in H$ such that $\overline{T_{1}(K)}$ is compact and every $T_{j}$ (except $T_{1}$ ) in the family $H$ is nonexpansive, then $H$ has a common fixed point in $K$.

Proof. Let $T_{1}, T_{2}$ and $T_{3} \in H$ and let $\Gamma$ be the set of all nonempty closed bounded convex subsets $A$ of $K$ such that $T_{1}(A) \subset A, T_{2}(A) \subset A$, and $T_{3}(A) \subset A$ and $\overline{T_{1}(A)}$ is compact. On the set $\Gamma$, define a partial order by set inclusion; then we can find a minimal set $K_{0} \in \Gamma$.

As in the proof of Theorem 17, $T_{1}$ and $T_{2}$ have a nonempty compact common fixed point set $F=F\left(T_{1}, T_{2}\right)$ in $K_{0}$ satisfying $T_{1}(F)=F$ and $T_{2}(F)=F$. Since $\left(T_{3}, T_{1}\right)$ and $\left(T_{3}, T_{2}\right)$ are Banach operator pairs, we have $T_{3}(F) \subset F$. Using Zorn's lemma, there exists a minimal nonempty compact subset $M$ of $K_{0}$ which satisfies $T_{1}(M)=M, T_{2}(M)=$ $M$, and $T_{3}(M) \subset M$. Using an argument similar to that in Theorem 17, we can show that $T_{3}(M)=M$. If $M$ reduces to a point, then $F\left(T_{1}, T_{2}, T_{3}\right) \neq \emptyset$. If $M$ has at least two different points, then, by Lemma 16 , this contradicts the minimality of $K_{0}$. Therefore we obtain that $K_{0}$ is a singleton and $F\left(T_{1}, T_{2}, T_{3}\right) \neq \emptyset$.

For any finite maps $T_{j} \in H, j=1,2, \ldots, n$, we have by induction that $F\left(T_{1}, T_{2}, \ldots, T_{n}\right) \neq \emptyset$. We now let $\theta=$ $\left\{F\left(T_{1}, T\right): T \in H\right\}$. Thus for any $T \in H, F\left(T_{1}, T\right)$ is a nonempty compact set, and for each $T_{j} \in H, j=2, \ldots, n$, we have

$$
\bigcap_{j=2}^{n} F\left(T_{1}, T_{j}\right)=F\left(T_{1}, T_{2}, \ldots, T_{n}\right) \neq \emptyset .
$$

This implies that the set family $\theta$ has the finite intersect property. Thus,

$$
\bigcap_{T \in H} F\left(T_{1}, T\right) \neq \emptyset
$$

Therefore the family $H$ has a common fixed point in $K$.

\section{Applications}

Let $K$ be a subset of the normed space $E$ and $\widehat{x} \in E$; then the distance of a point $\widehat{x}$ to the subset $K$ is defined by

$$
\operatorname{dist}(\widehat{x}, K)=\inf \{\|y-\widehat{x}\|: y \in K\} .
$$

The set of best approximants of a point $\widehat{x}$ in $K$ is denoted by $P_{K}(\widehat{x})$ and defined by

$$
P_{K}(\widehat{x})=\{y \in K:\|y-\widehat{x}\|=\operatorname{dist}(\widehat{x}, K)\} .
$$

It is well known that $P_{K}(\widehat{x})$ is always a bounded subset of $K$ and is a closed and convex set if $K$ is so. Also, if $K$ is compact, then $P_{K}(\widehat{x})$ is nonempty. For more details, we refer to [17].

Let $\Omega_{0}$ denote the class of closed convex subsets of $E$ containing 0 . For $K \in \Omega_{0}$ and $\widehat{x} \in E$, let

$$
K_{\widehat{x}}=\{x \in K:\|x\| \leq 2\|\widehat{x}\|\} .
$$

It is clear that $P_{K}(\widehat{x}) \subset K_{\widehat{x}} \in \Omega_{0}$.

The following result provides a partial solution of an existence problem of approximation theory in the following result (see also [14]).

Theorem 23. Let $E$ be a Banach space and let $T$ be a self-map of $E$ with $\widehat{x} \in F(T)$ and $K \in \Omega_{0}$ such that $T\left(K_{\widehat{x}}\right) \subset K$. Assume that $T$ satisfies condition $(C)$ on $K_{\widehat{x}} \cup\{\widehat{x}\}$ and $\overline{T\left(K_{\widehat{x}}\right)}$ is compact. Then the set of best approximations $P_{K}(\hat{x})$ is nonempty.

Proof. Without loss of generality we may assume that $\widehat{x} \in E \backslash$ $K$. If $x \in K \backslash K_{\widehat{x}}$, then

$$
\begin{aligned}
\|x-\widehat{x}\| & \geq\|x\|-\|\widehat{x}\| \\
& >2\|\widehat{x}\|-\|\widehat{x}\| \\
& =\|\widehat{x}\|
\end{aligned}
$$




$$
\begin{aligned}
& \geq \operatorname{dist}\left(\widehat{x}, K_{\widehat{x}}\right) \\
& \geq \operatorname{dist}(\widehat{x}, K) .
\end{aligned}
$$

As a result

$$
\operatorname{dist}\left(\widehat{x}, K_{\widehat{x}}\right)=\operatorname{dist}(\widehat{x}, K) .
$$

Since $\overline{T\left(K_{\widehat{x}}\right)}$ is compact, we can find $y \in \overline{T\left(K_{\widehat{x}}\right)}$ such that

$$
\operatorname{dist}\left(\widehat{x}, \overline{T\left(K_{\widehat{x}}\right)}\right)=\|y-\widehat{x}\|,
$$

and so by Lemma 14

$$
\begin{aligned}
\operatorname{dist}\left(\widehat{x}, K_{\widehat{x}}\right) & =\operatorname{dist}(\widehat{x}, K) \\
& \leq \operatorname{dist}\left(\widehat{x}, \overline{T\left(K_{\widehat{x}}\right)}\right) \\
& \leq\|T x-T \widehat{x}\| \\
& \leq\|x-\widehat{x}\|
\end{aligned}
$$

for all $x \in K_{\widehat{x}}$. Hence

$$
\operatorname{dist}(\widehat{x}, K)=\operatorname{dist}\left(\widehat{x}, K_{\widehat{x}}\right)=\operatorname{dist}\left(\widehat{x}, \overline{T\left(K_{\widehat{x}}\right)}\right)=\|y-\hat{x}\|
$$

and thus $y \in P_{K}(\widehat{x})$.

The following is an application of Theorem 17 to invariant approximations for convex sets.

Theorem 24. Let $E$ be a Banach space, I and T self-maps of $E$ with $\hat{x} \in F(I, T)$, and $K \in \Omega_{0}$ with $I\left(K_{\widehat{x}}\right) \subset K$ and $T\left(K_{\widehat{x}}\right) \subset K$. If $(I, T)$ is a Banach operator pair on $K_{\hat{x}}$, both $I$ and T are maps satisfying condition $(C)$ on $K_{\widehat{x}} \cup\{\hat{x}\}, I$ is nonexpansive, and $\overline{T\left(K_{\widehat{x}}\right)}$ is compact, then $F(I, T) \cap P_{K}(\widehat{x}) \neq \emptyset$.

Proof. By Theorem 23, $P_{K}(\widehat{x})$ is a nonempty. Since $K$ is closed and convex, then $P_{K}(\widehat{x})$ is a closed convex set. We now show that $P_{K}(\widehat{x})$ is $T$-invariant. Let $y \in P_{K}(\widehat{x})$. Then $\|y-\widehat{x}\|=$ $\operatorname{dist}(\widehat{x}, K)$. Since $T$ satisfies condition $(\mathrm{C})$ on $K_{\widehat{x}} \cup\{\widehat{x}\}$, by Lemma 14, we obtain that

$$
\|T(y)-\widehat{x}\|=\|T(y)-T(\widehat{x})\| \leq\|y-\hat{x}\|,
$$

and so

$$
\operatorname{dist}(\widehat{x}, K) \leq\|T(y)-\hat{x}\| \leq\|y-\hat{x}\|=\operatorname{dist}(\widehat{x}, K) .
$$

This implies that $T(y) \in P_{K}(\widehat{x})$. Consequently, we have $T\left(P_{K}(\widehat{x})\right) \subset P_{K}(\widehat{x})$, and, similarly, we can prove that $I\left(P_{K}(\widehat{x})\right)$ $\subset P_{K}(\widehat{x})$. Since $\overline{T\left(P_{K}(\widehat{x})\right)} \subset \overline{T\left(K_{\widehat{x}}\right)}$ and $\overline{T\left(K_{\widehat{x}}\right)}$ is compact, we have that $\overline{T\left(P_{K}(\widehat{x})\right)}$ is compact. Now, Theorem 17 guarantees that $F(I, T) \cap P_{K}(\widehat{x}) \neq \emptyset$.

\section{Acknowledgments}

The authors are grateful to the referees for their valuable suggestions. This article was funded by the Deanship of Scientific Research (DSR), King Abdulaziz University, Jeddah. The first and third authors acknowledge with thanks DSR for financial support. The second author is grateful to Chiang Mai University for the financial support.

\section{References}

[1] G. Jungck, "Commuting mappings and fixed points," The American Mathematical Monthly, vol. 83, no. 4, pp. 261-263, 1976.

[2] S. Sessa and B. Fisher, "On fixed points of weakly commuting mappings in compact metric spaces," Jñānābha, vol. 15, pp. 7991, 1985.

[3] R. P. Pant, "Common fixed points of noncommuting mappings," Journal of Mathematical Analysis and Applications, vol. 188, no. 2, pp. 436-440, 1994.

[4] N. Shahzad, "Invariant approximations and $R$-subweakly commuting maps," Journal of Mathematical Analysis and Applications, vol. 257, no. 1, pp. 39-45, 2001.

[5] J. Chen and Z. Li, "Common fixed-points for Banach operator pairs in best approximation," Journal of Mathematical Analysis and Applications, vol. 336, no. 2, pp. 1466-1475, 2007.

[6] M. A. Al-Thagafi and N. Shahzad, "Banach operator pairs, common fixed-points, invariant approximations, and *nonexpansive multimaps," Nonlinear Analysis. Theory, Methods \& Applications A, vol. 69, no. 8, pp. 2733-2739, 2008.

[7] G. Jungck, "Compatible mappings and common fixed points," International Journal of Mathematics and Mathematical Sciences, vol. 9, no. 4, pp. 771-779, 1986.

[8] G. Jungck and H. K. Pathak, "Fixed points via 'biased maps," Proceedings of the American Mathematical Society, vol. 123, no. 7, pp. 2049-2060, 1995.

[9] M. A. Al-Thagafi and N. Shahzad, "Generalized $I$-nonexpansive selfmaps and invariant approximations," Acta Mathematica Sinica, vol. 24, no. 5, pp. 867-876, 2008.

[10] J. Chen and Z. Li, "Banach operator pair and common fixed points for nonexpansive maps," Nonlinear Analysis. Theory, Methods \& Applications A, vol. 74, no. 10, pp. 3086-3090, 2011.

[11] T. Suzuki, "Fixed point theorems and convergence theorems for some generalized nonexpansive mappings," Journal of Mathematical Analysis and Applications, vol. 340, no. 2, pp. 1088-1095, 2008.

[12] S. Dhompongsa, W. Inthakon, and A. Kaewkhao, "Edelstein's method and fixed point theorems for some generalized nonexpansive mappings," Journal of Mathematical Analysis and Applications, vol. 350, no. 1, pp. 12-17, 2009.

[13] S. Dhompongsa and A. Kaewcharoen, "Fixed point theorems for nonexpansive mappings and Suzuki-generalized nonexpansive mappings on a Banach lattice," Nonlinear Analysis. Theory, Methods \& Applications A, vol. 71, no. 11, pp. 5344-5353, 2009.

[14] N. Shahzad and G. Bassindowa, "Fixed point theorems for Suzuki-generalized nonexpansive mappings with applications," Journal of Nonlinear and Convex Analysis, vol. 13, no. 4, pp. 657666, 2012.

[15] R. DeMarr, "Common fixed points for commuting contraction mappings," Pacific Journal of Mathematics, vol. 13, pp. 1139-1141, 1963.

[16] M. S. Brodskii and D. P. Milman, "On the center of a convex set," Doklady Akademii Nauk SSSR, vol. 59, pp. 837-840, 1948 (Russian).

[17] S. Singh, B. Watson, and P. Srivastava, Fixed Point Theory and Best Approximation, The KKM-Map Principle, vol. 424, Kluwer Academic Publishers, Dordrecht, The Netherlands, 1997. 


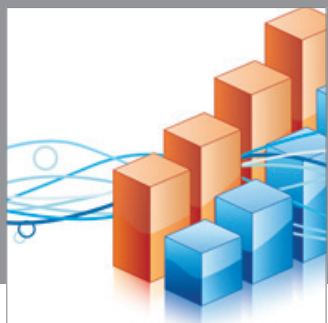

Advances in

Operations Research

mansans

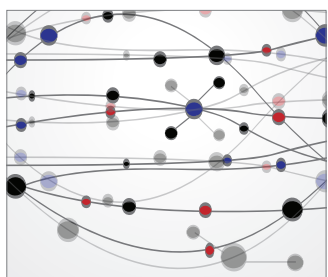

The Scientific World Journal
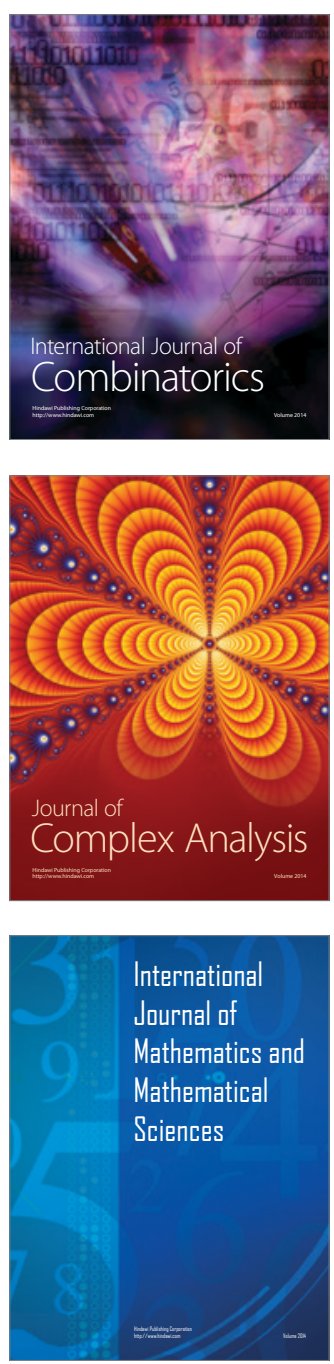
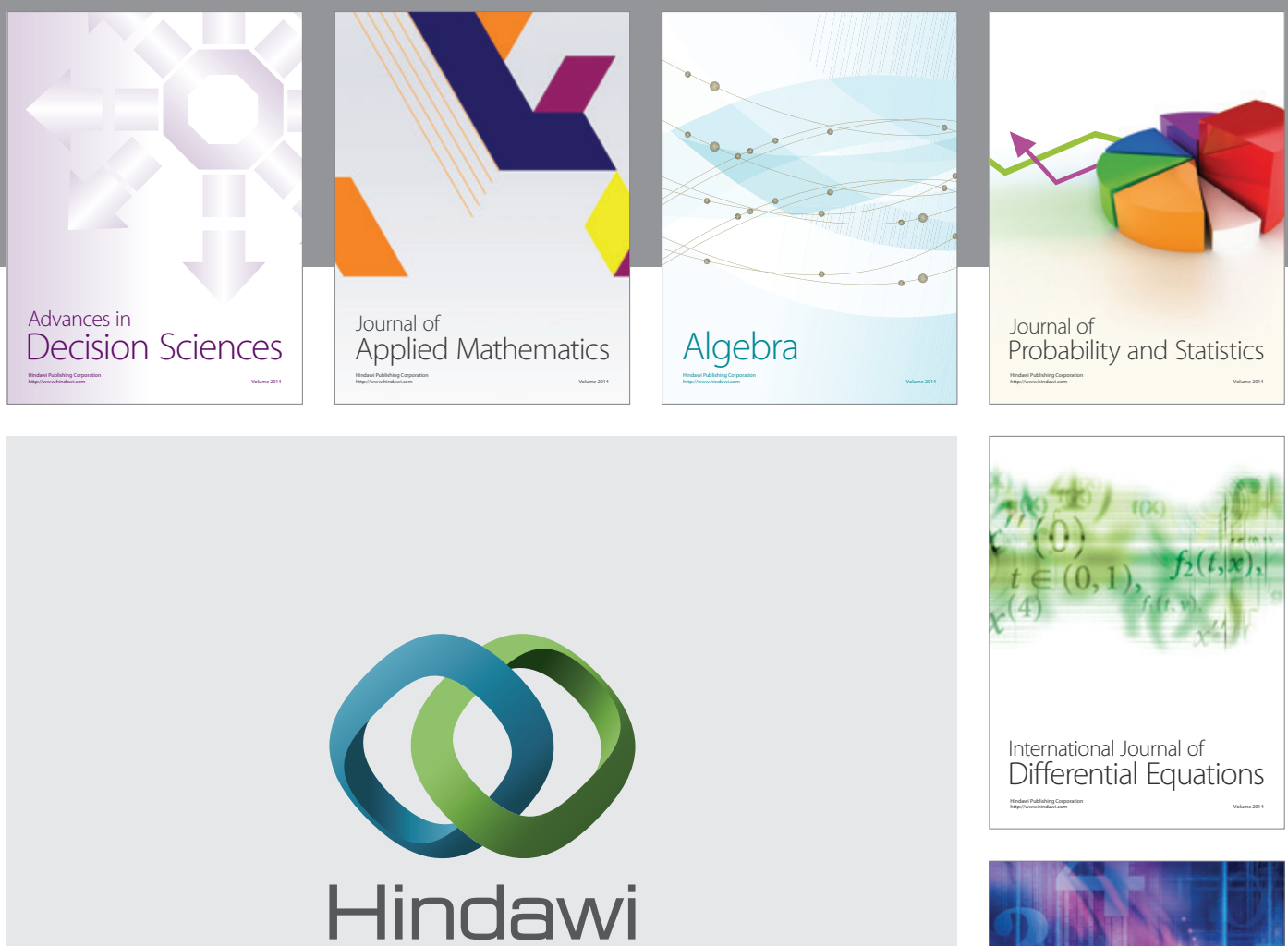

Submit your manuscripts at http://www.hindawi.com
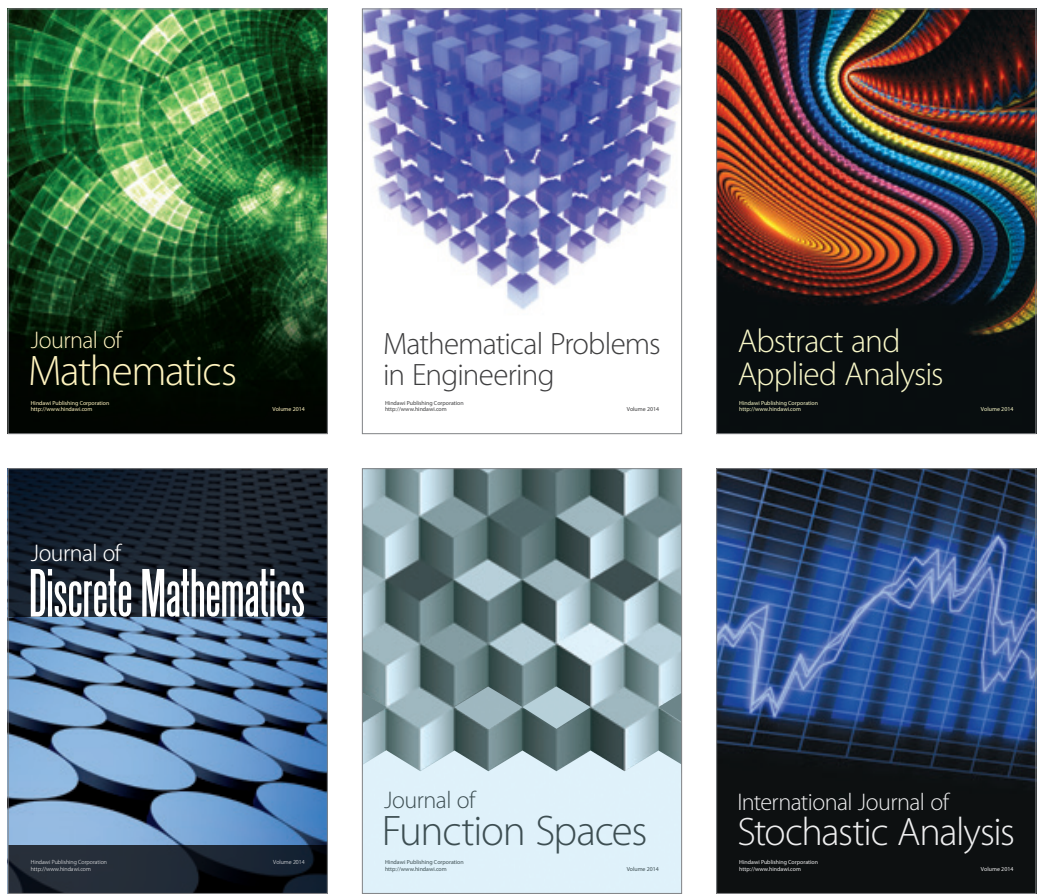

Journal of

Function Spaces

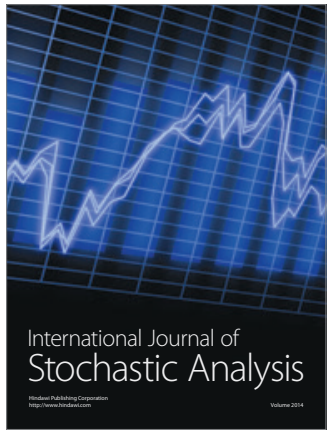

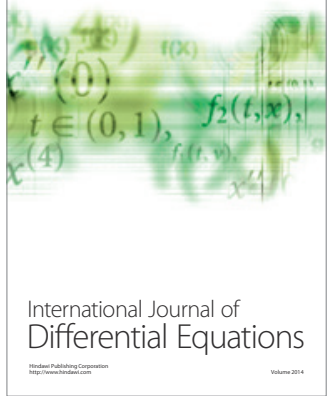
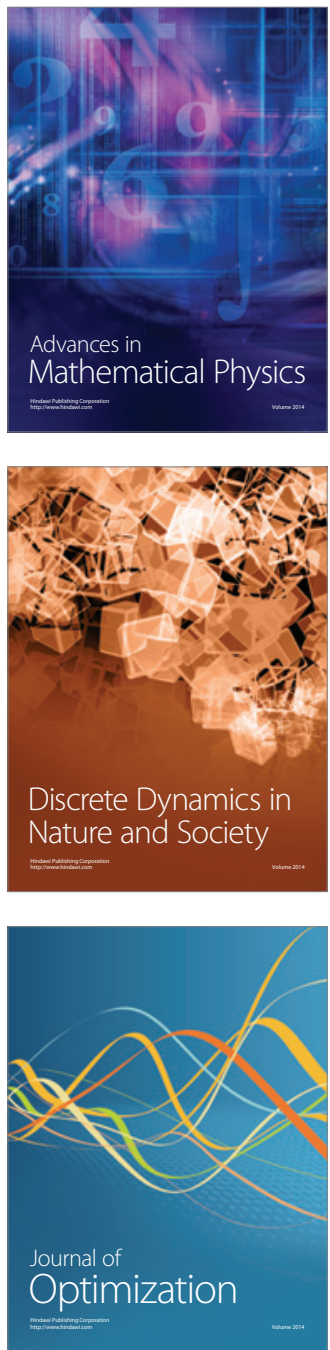